\title{
Probabilidade de transição e condicional para chuva mensal e extremos diários em Santa Catarina
}

\author{
Rosandro Boligon Minuzzi $i^{1 *}$ \\ 10.1590/0034-737X201663060005
}

\section{RESUMO}

Este estudo objetivou obter matrizes de probabilidade, de transição e condicional, da chuva mensal, para o Estado de Santa Catarina, como forma de auxiliar a previsão climática. Foram utilizados dados diários de chuva do período de 1991 a 2013, de nove estações localizadas em diferentes regiões climáticas do Estado de Santa Catarina. Para obter as matrizes de probabilidades, as chuvas mensais foram divididas nas seguintes categorias: abaixo (-1), dentro (0) e acima (1) da normal climatológica. O enquadramento dos valores de chuva mensal nas referidas categorias foi baseado nos quantis $\mathrm{Q}_{0,35}$ e $\mathrm{Q}_{0,65}$. Quando a chuva observada fica abaixo da normal climatológica (categoria -1) há maior probabilidade de a chuva do mês seguinte passar para a categoria 1 em seis regiões, representadas pelos municípios de Vargem Bonita (40\%), Saudades (47\%), Curitibanos (36\%), Lages (40\%), Trombudo Central (40\%) e Araranguá (45\%). Situação semelhante ocorre quando, em dado mês, a chuva observada fica acima da normal climatológica, ou seja, há maior probabilidade de permanecer na categoria 1. Os valores probabilísticos mostram que as matrizes de probabilidade, de transição e condicional, podem ser usadas como auxílio para as previsões mensais e de eventos extremos diários de chuva para Santa Catarina.

Palavras-chave: climatologia; cadeia de Markov; eventos extremos.

\section{ABSTRACT}

\section{Transition and conditional probability for monthly and extreme daily rainfall in Santa Catarina}

This study aimed to obtain probability matrices, of transition and conditional, of monthly rainfall for the state of Santa Catarina, as an aid to climate forecast. We used daily rainfall data of the period 1991-2013 from nine stations located in different climatic regions of Santa Catarina. For matrices of probabilities, monthly rainfalls were divided into the following categories: below (-1), within (0), and above (1) climatology. The framework of the values of monthly rainfall in these categories was based on quantiles $\mathrm{Q}_{0.35}$ and $\mathrm{Q}_{0.65}$. When the rainfall is below the climatology (category $-1)$, there is a greater probability that the rainfall in the following month changes to category 1 in six regions, represented by the cities of Vargem Bonita (40\%), Saudades (47\%), Curitibanos (36\%), Lages (40\%), Trombudo Central (40\%), and Araranguá (45\%). A similar situation occurs when, in a given month, the observed rainfall is above the climatology, that is, it is more likely to remain in category 1 . The probability values show that the matrices of transition and conditional probabilities can be used as an aid in monthly forecasts and daily extreme events of rainfall for Santa Catarina.

Key words: climatology; Markov chain; extreme events.

\footnotetext{
Recebido para publicação em 12/12/2014 e aprovado em 20/07/2016.

${ }^{1}$ Departamento de Engenharia Rural, Universidade Federal de Santa Catarina, Florianópolis, Santa Catarina, Brasil. rbminuzzi@hotmail.com

*Autor para correspondência: rbminuzzi@ hotmail.com
} 


\section{INTRODUÇÃO}

As chuvas em Santa Catarina apresentam um regime bem distribuído durante todo o ano, permitindo que a agricultura seja desenvolvida em qualquer época do ano, desde que não haja impedimento por outro elemento climático. A classificação climática por Köppen atesta esse regime de chuvas, mas divide o Estado em dois tipos de clima, em função da grande variabilidade térmica anual. A região central apresenta verões brandos e invernos rigorosos $(\mathrm{Cfb})$, enquanto, no restante do Estado (oeste e litoral), os verões são rigorosos e os invernos são brandos (Cfa) (Vianello \& Alves, 1991). De acordo com dados médios obtidos de 2002 a 2012 (IBGE, 2014), o Estado tem destaque na produção agrícola nacional, como no cultivo da maçã e da cebola ( $1^{\mathrm{a}}$ posição), de pêssego ( $2^{\underline{a}}$ posição), de trigo ( $3^{\underline{a}}$ posição), de alho (4 $4^{\mathbf{a}}$ posição) e de arroz e de uva ( $5^{\mathbf{a}}$ posição). Mesmo sem afetar essa relevância no cenário agrícola, eventualmente ocorrem reduções de produtividade decorrentes direta ou indiretamente da variabilidade pluviométrica, tornando a agricultura uma atividade de risco.

Grimm (2009) destaca que, tanto no verão quanto nas estações de transição, os Complexos Convectivos de Mesoescala (CCM) são frequentes e respondem por grande parte da precipitação total da região sul do Brasil. Acrescenta que, em grande parte de Santa Catarina, predomina o regime de monções de verão, com o máximo de chuvas, de 160 a $180 \mathrm{~mm}$ no trimestre de dezembro a fevereiro.

Neste contexto, a previsão climática apresenta-se como uma ferramenta fundamental para não somente agropecuaristas, mas também o setor elétrico, abastecimento de água, seguradoras rurais e cooperativas. Uma previsão climática mais detalhada resulta numa decisão mais precisa para atividades econômicas e humanas, algo que, em muitas situações, a habitual previsão climática trimestral pode não satisfazer. O estudo de Fox et al. (1999) mostrou a importância econômica da previsão de chuvas para o cultivo do trigo em dois locais na Inglaterra. Berlato \& Fontana (2001) recorreram a resultados com várias culturas temporárias (soja, milho, arroz, trigo e outros cereais de inverno) no Rio Grande do Sul, para mostrarem que a previsão climática constitui inestimável informação para o manejo agrícola, à medida que propicia minimização de riscos em condições desfavoráveis e melhor previsão das situações favoráveis.

No decorrer dos anos, os pesquisadores têm desenvolvido considerável competência em modelagem e simulação de chuvas. Um dos métodos empregados é o dos modelos Markovianos, que vêm sendo utilizados nos processos climatológicos que apresentam modelagem sequencial. Wilks (2006) atesta essa informação, destacando que a Cadeia de Markov tem sido usada para caracterizar a transição entre os meses abaixo, dentro e acima das normais climatológicas, como definido pelo Centro de Previsão Climática dos Estados Unidos (CPC). Outro exemplo de aplicação da cadeia de Markov é apresentado por Soares et al. (1998), em estudo objetivando caracterizar a periodicidade das precipitações anuais para determinar a probabilidade de se ter, em um período especificado, um número de anos com determinada precipitação anual, como subsídios para o planejamento florestal, no município de Inhambupe, Estado da Bahia.

Este estudo teve como objetivo obter matrizes de probabilidade de transição e condicional da chuva mensal para o Estado de Santa Catarina.

\section{MATERIAL E MÉTODOS}

Foram utilizados dados pluviométricos diários do período de 1991 a 2013 de nove estações hidrológicas convencionais do Estado de Santa Catarina (Figura 1), oriundos do Sistema de Informações Hidrológicas (HidroWeb) da Agência Nacional de Águas (ANA, 2014), de forma a representar, cada uma, a maioria das regiões climaticamente homogêneas do Estado, de acordo com Braga \& Ghellre (1999).

Para obter a matriz das probabilidades de transição $\left(\mathrm{P}_{\mathrm{ij}}\right)$, na cadeia de Markov, as chuvas mensais foram divididas nas seguintes categorias: abaixo (-1), dentro (0) e acima (1) da climatologia. Vianello \& Alves (1991) apresentam diferentes definições encontradas na literatura, para clima, sendo todas válidas, porém variáveis, segundo o ponto de vista do autor e as finalidades propostas nas pesquisas. Neste estudo, considera-se a climatologia de chuva como sendo o valor que caracteriza o estado médio da atmosfera dessa variável em dado local.

O enquadramento dos valores de chuva (C) mensal nas referidas categorias foi baseado nos quantis $\mathrm{Q}_{0,35} \mathrm{e}$ $\mathrm{Q}_{0,65}$, conforme consta (Xavier, 2001):

Categoria -1 (abaixo da climatologia): $\mathrm{C} \leq \mathrm{Q}_{0,35}$ Categoria 0 (dentro da climatologia): $\mathrm{Q}_{0,35}<\mathrm{C}<\mathrm{Q}_{0,65}$ Categoria 1 (acima da climatologia): $\mathrm{C} \geq \mathrm{Q}_{0,65}$

Assim, os valores que enquadram a chuva nas referidas categorias, para cada uma das estações utilizadas no estudo, constam na Tabela 1.

Dessa forma, a matriz das probabilidades de transição $\left(\mathrm{P}_{\mathrm{ij}}\right)$ ficou assim definida:

\begin{tabular}{lccc}
\hline Categorias & $\mathbf{- 1}$ & $\mathbf{0}$ & $\mathbf{1}$ \\
\hline-1 & $\mathrm{P}_{-1-1}$ & $\mathrm{P}_{-10}$ & $\mathrm{P}_{-11}$ \\
0 & $\mathrm{P}_{0-1}$ & $\mathrm{P}_{00}$ & $\mathrm{P}_{01}$ \\
1 & $\mathrm{P}_{1-1}$ & $\mathrm{P}_{10}$ & $\mathrm{P}_{11}$ \\
\hline
\end{tabular}

em que,

$\mathrm{P}_{-1-1}=$ probabilidade de a chuva estar na categoria -1 , no mês $t$, e permanecer na categoria -1 , no mês $t+1$ 
$\mathrm{P}_{-10}=$ probabilidade de a chuva estar na categoria -1 , no mês $t$, e passar para a categoria 0 , no mês $t+1$

.....

$\mathrm{P}_{11}=$ probabilidade de a chuva estar na categoria 1 , no mês $\mathrm{t}$, e permanecer na categoria 1 , no mês $\mathrm{t}+1$.

As probabilidades de transição $\left(\mathrm{P}_{-1-1}\right.$ a $\left.\mathrm{P}_{11}\right)$ foram calculadas pelas seguintes expressões:

Para $\mathrm{i}=\mathrm{j}, \mathrm{i}$ e $\mathrm{j}=-1,0$ e 1

$\mathrm{P}_{\mathrm{ij}}=\underline{\mathrm{n}}$,

$\mathrm{N}$

em que $\mathrm{n}$ é o número de meses em que a chuva mensal mudou da categoria i, no mês t, para a categoria $j$, no mês $\mathrm{t}+1$, e $\mathrm{N}$ é o número de meses de chuva mensal ocorridos na categoria $i$.

Considerando-se um período de planejamento de dois meses, a probabilidade condicional de ocorrer dois (2), um (1) e zero (0) meses de chuva mensal, nas categorias i (i = 1,0 e 1), foi calculada por:

$\mathrm{P}(2$ meses na categoria i)

$\mathrm{P}_{\mathrm{X}} \cdot \mathrm{P}_{\mathrm{i} / \mathrm{X}} \cdot \mathrm{P}_{\mathrm{i} / \mathrm{i}}$

$\mathrm{P}$ (1 mês na categoria i)

$\mathrm{P}_{\mathrm{X}} \cdot \mathrm{P}_{\mathrm{X} / \mathrm{X}} \cdot \mathrm{P}_{\mathrm{i} / \mathrm{X}}$

$\mathrm{P}(0$ mês na categoria i)

$$
\begin{aligned}
& \mathrm{P}_{\mathrm{i}} \cdot \mathrm{P}_{\mathrm{i} / \mathrm{i}} \cdot \mathrm{P}_{\mathrm{X} / \mathrm{i}}+\mathrm{P}_{\mathrm{i}} \cdot \mathrm{P}_{\mathrm{X} / \mathrm{i}} \cdot \mathrm{P}_{\mathrm{i} / \mathrm{X}}+ \\
& \mathrm{P}_{\mathrm{i}} \cdot \mathrm{P}_{\mathrm{X} / \mathrm{i}} \cdot \mathrm{P}_{\mathrm{X} / \mathrm{X}}+\mathrm{P}_{\mathrm{X}} \cdot \mathrm{P}_{\mathrm{i} / \mathrm{x}} \cdot \mathrm{P}_{\mathrm{X} / \mathrm{i}}+ \\
& \mathrm{P}_{\mathrm{X}} \cdot\left(\mathrm{P}_{\mathrm{X} / \mathrm{X}}\right)^{2}
\end{aligned}
$$

em que,

$\mathrm{X}=$ não estar na categoria $\mathrm{i}$;

$\mathrm{P}_{\mathrm{X}}=1-\mathrm{P}_{\mathrm{i}}=$ probabilidade de não estar na categoria $\mathrm{i}$;

$\mathrm{P}_{\mathrm{i}}=\mathrm{n} / \mathrm{N}=$ probabilidade de estar na categoria $\mathrm{i}$;

$\mathrm{P}_{\mathrm{X} / \mathrm{i}}=1-\mathrm{P}_{\mathrm{i} / \mathrm{i}}=$ probabilidade de não estar na categoria $\mathrm{i}$, dado que estava na categoria $\mathrm{i}$;
$\mathrm{P}_{\mathrm{i} / \mathrm{i}}=$ probabilidade de estar na categoria $\mathrm{i}$, dado que estava na categoria $\mathrm{i}$;

$\mathrm{P}_{\mathrm{i} / \mathrm{X}}=\left(\mathrm{P}_{\mathrm{i}} \cdot \mathrm{P}_{\mathrm{X} / \mathrm{i}}\right) / \mathrm{P}_{\mathrm{X}}=$ probabilidade de estar na categoria $\mathrm{i}$, dado que não estava na categoria $\mathrm{i}$; $\mathrm{e}$

$\mathrm{P}_{\mathrm{X} / \mathrm{X}}=1-\mathrm{P}_{\mathrm{i} / \mathrm{X}}=$ probabilidade de não estar na categoria $\mathrm{i}$, dado que não estava na categoria $i$.

Foi realizada uma associação das ocorrências e, ou, ausência mensal de eventos extremos diários de chuva, com cada uma das três categorias, com o uso da distribuição de frequência relativa. Para a definição de um evento extremo diário de chuva, foi considerado o dia com valor igual ou maior ao obtido pelo percentil 0,95 de cada estação (Tabela 1). Para o cálculo desse valor, foram desconsiderados os dias com registros diários de chuva inferiores a $0,9 \mathrm{~mm}$, pois, geralmente, uma quantidade inferior a esta não se infiltra no solo. Para a organização das séries mensais de chuva e a determinação dos quantis foi utilizada a planilha Excel, enquanto as probabilidades de transição e condicional foram obtidas com o software PAST 3.12.

\section{RESULTADOS E DISCUSSÃO}

As informações da Tabela 2 mostram as probabilidades de a chuva mensal estar abaixo (-1), dentro (0) ou acima (1) da climatologia, com base na categoria de chuva observada no mês anterior. Como exemplo, se em setembro a chuva na região de Vargem Bonita esteve abaixo da climatologia (inferior a $136 \mathrm{~mm}$, conforme Tabela 1), há uma maior probabilidade $(40 \%)$ de que a chuva em outubro fique acima da climatologia, ou com valor superior a 219 mm. Outra forma de interpretar os resultados, envolve a

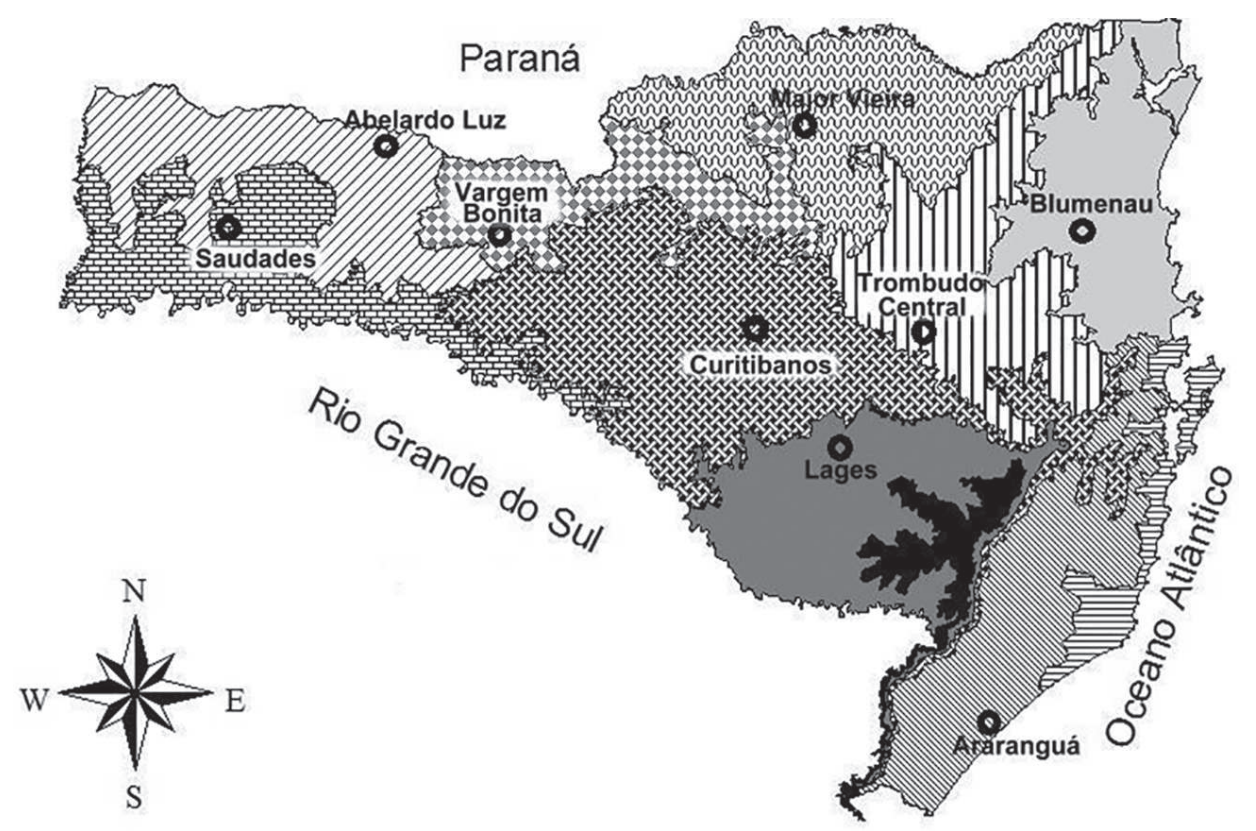

Figura 1: Localização geográfica das estações hidrológicas nas diferentes regiões climáticas de Santa Catarina e utilizadas no estudo. Rev. Ceres, Viçosa, v. 63, n.6, p. 775-781, nov/dez, 2016 
probabilidade de a chuva ficar dentro da climatologia, juntamente com a probabilidade indicada do estado -1 ou 1 . Assim, no referido exemplo para Vargem Bonita, poder-seia afirmar que a chuva em outubro tem $70 \%$ de probabilidade de ficar na média (30\%) a acima (40\%) da climatologia, ou $70 \%$ de chover mais do que $174 \mathrm{~mm}$.

Percebe-se que quando a chuva observada fica abaixo da climatologia (categoria -1) há maior probabilidade de a chuva no mês seguinte passar para a categoria 1 em seis regiões, representadas pelos municípios de Vargem Bonita (40\%), Saudades (47\%), Curitibanos (36\%), Lages (40\%), Trombudo Central (40\%) e Araranguá (45\%). Em Blumenau e Abelardo Luz, a probabilidade é maior de a chuva ficar abaixo da climatologia (42\%), enquanto em Major Vieira, há praticamente uma igualdade entre as probabilidades obtidas para as categorias -1 e 1 .

Situação semelhante ocorre quando em dado mês a chuva observada fica acima da climatologia, ou seja, há maior probabilidade de ela permanecer na categoria 1 na maioria dos municípios analisados. A exceção foi em Lages onde a probabilidade é maior de a chuva ficar dentro da climatologia (40\%).

Os percentuais obtidos nas situações em que a chuva observada fica dentro da climatologia são relativamente mais variados e, em algumas situações, tornam a decisão incerta quanto a sua utilização para previsão de clima, como em Major Vieira e Saudades, havendo nesta última, probabilidades iguais (38\%) para que a chuva fique abaixo e acima da climatologia. Isto provavelmente se deve pela grande variabilidade espaço-temporal da chuva, associado com o menor intervalo que envolve os valores de chuva da categoria 0. Dias \& Silva (2009) atestam esta informação quando destacam que apesar de o regime de chuvas de um determinado local ser fator dominante na definição do clima local, as chuvas são o resultado final de uma série de eventos com escalas de tempo e espaço bastante

Tabela 1: Valores arredondados mensais de chuva (mm) obtidos pelos quantis $0,35\left(\mathrm{Q}_{0,35}\right)$ e $0,65\left(\mathrm{Q}_{0,65}\right)$ e os limites que definem os eventos extremos (Ee) diários de chuva para os municípios de Vargem Bonita (VB), Abelardo Luz (AL), Saudades (Sau), Curitibanos (Cur), Lages (Lag), Trombudo Central (TC), Araranguá (Ara), Major Vieira (MV) e Blumenau (Blu)

\begin{tabular}{|c|c|c|c|c|c|c|c|c|c|}
\hline & \multicolumn{2}{|c|}{ Jan } & \multicolumn{2}{|c|}{$\mathrm{Fev}$} & \multicolumn{2}{|c|}{ Mar } & \multicolumn{2}{|c|}{ Abr } & \multirow[b]{2}{*}{$\mathrm{Ee}$} \\
\hline & $\mathrm{Q}_{0,35}$ & $\mathrm{Q}_{0,65}$ & $\mathrm{Q}_{0,35}$ & $\mathrm{Q}_{0,65}$ & $\mathrm{Q}_{0,35}$ & $\mathrm{Q}_{0,65}$ & $\mathrm{Q}_{0,35}$ & $\mathrm{Q}_{0,65}$ & \\
\hline VB & 154 & 218 & 140 & 212 & 112 & 145 & 98 & 169 & 56 \\
\hline $\mathrm{AL}$ & 130 & 206 & 130 & 193 & 104 & 181 & 109 & 163 & 50 \\
\hline $\mathrm{Sau}$ & 129 & 182 & 116 & 169 & 86 & 157 & 107 & 162 & 62 \\
\hline Cur & 128 & 181 & 119 & 180 & 83 & 128 & 65 & 124 & 46 \\
\hline Lag & 120 & 192 & 136 & 169 & 92 & 135 & 79 & 124 & 45 \\
\hline $\mathrm{TC}$ & 140 & 196 & 129 & 173 & 96 & 130 & 55 & 108 & 41 \\
\hline Ara & 102 & 176 & 127 & 176 & 115 & 174 & 71 & 99 & 46 \\
\hline MV & 128 & 183 & 127 & 177 & 93 & 145 & 63 & 111 & 53 \\
\hline \multirow[t]{3}{*}{ Blu } & 172 & 228 & 162 & 236 & 135 & 180 & 75 & 114 & 46 \\
\hline & \multicolumn{2}{|c|}{ Mai } & \multicolumn{2}{|c|}{ Jun } & \multicolumn{2}{|c|}{ Jul } & \multicolumn{2}{|c|}{ Ago } & \\
\hline & $\mathrm{Q}_{0,35}$ & $\mathrm{Q}_{0,65}$ & $\mathrm{Q}_{0,35}$ & $\mathrm{Q}_{0,65}$ & $\mathrm{Q}_{0,35}$ & $\mathrm{Q}_{0,65}$ & $\mathrm{Q}_{0,35}$ & $\mathrm{Q}_{0,65}$ & \\
\hline VB & 81 & 169 & 102 & 161 & 95 & 156 & 108 & 182 & \\
\hline $\mathrm{AL}$ & 92 & 172 & 116 & 162 & 103 & 150 & 101 & 179 & \\
\hline Sau & 83 & 162 & 83 & 162 & 98 & 158 & 80 & 142 & \\
\hline Cur & 66 & 126 & 69 & 125 & 75 & 133 & 75 & 142 & \\
\hline Lag & 68 & 124 & 82 & 136 & 90 & 151 & 91 & 163 & \\
\hline $\mathrm{TC}$ & 57 & 117 & 66 & 108 & 81 & 109 & 75 & 123 & \\
\hline Ara & 56 & 89 & 63 & 101 & 65 & 107 & 74 & 124 & \\
\hline MV & 64 & 118 & 89 & 153 & 72 & 140 & 69 & 134 & \\
\hline \multirow[t]{3}{*}{ Blu } & 62 & 105 & 78 & 114 & 82 & 115 & 54 & 110 & \\
\hline & \multicolumn{2}{|c|}{ Set } & \multicolumn{2}{|c|}{ Out } & \multicolumn{2}{|c|}{ Nov } & \multicolumn{2}{|c|}{ Dez } & \\
\hline & $Q_{0,35}$ & $Q_{0,65}$ & $\mathrm{Q}_{0,35}$ & $\mathrm{Q}_{0,65}$ & $\mathrm{Q}_{0,35}$ & $\mathrm{Q}_{0,65}$ & $\mathrm{Q}_{0,35}$ & $\mathrm{Q}_{0,65}$ & \\
\hline $\mathrm{VB}$ & 136 & 199 & 174 & 219 & 113 & 185 & 136 & 198 & \\
\hline $\mathrm{AL}$ & 134 & 196 & 159 & 248 & 103 & 172 & 129 & 177 & \\
\hline Sau & 86 & 150 & 127 & 194 & 99 & 161 & 111 & 143 & \\
\hline Cur & 101 & 165 & 130 & 186 & 92 & 147 & 110 & 168 & \\
\hline Lag & 121 & 178 & 113 & 170 & 98 & 142 & 98 & 133 & \\
\hline $\mathrm{TC}$ & 110 & 152 & 117 & 178 & 87 & 129 & 97 & 167 & \\
\hline Ara & 91 & 154 & 85 & 131 & 85 & 129 & 79 & 131 & \\
\hline MV & 126 & 172 & 138 & 182 & 93 & 133 & 105 & 164 & \\
\hline Blu & 111 & 158 & 115 & 153 & 80 & 133 & 123 & 162 & \\
\hline
\end{tabular}

Rev. Ceres, Viçosa, v. 63, n.6, p. 775-781, nov/dez, 2016 
distintas, de forma que as causas de determinada chuva podem ser uma mistura de fatores locais e remotos.

No contexto geral, mesmo havendo diferentes regiões climáticas em Santa Catarina, a tendência dos resultados probabilísticos para cada uma das três categorias de chuva foi semelhante entre si. Azevedo et al. (1998) encontraram resultados satisfatórios quando objetivaram aferir um modelo de previsão de valores mínimo e máximo de chuva para a $2^{\text {a }}$ metade da estação chuvosa (20 de março a 30 de junho), com base na chuva totalizada na $1^{\mathrm{a}}$ metade desta estação ( $1^{\circ}$ de janeiro a 19 de março) para microrregiões do Ceará.

A matriz de probabilidade condicional, obtida para os municípios utilizados no estudo, é apresentada na Tabela 3. Num contexto geral, as maiores probabilidades para cada uma das três categorias de chuva é que estas ocorram ao menos uma vez a cada dois meses.

Para tornar uma informação mais proveitosa, pode-se recorrer ao raciocínio semelhante ao utilizado na análise das probabilidades de transição, ou seja, envolver a soma dos dois maiores valores obtidos entre as categorias de chuva. Seguindo este raciocínio, pode-se afirmar que em Saudades há $84 \%$ de probabilidade de que em dois meses seguidos observe-se chuva dentro da climatologia no máximo em apenas um mês (43\% para que este estado ocorra uma vez e $41 \%$ de que não seja observado em nenhum dos dois meses). Já para chuva acima da climatologia, há $76 \%$ de probabilidade de que esta categoria de chuva seja observada ao menos uma vez (32\% para que ocorra nos dois meses e $44 \%$ para que ocorra uma vez).

Tabela 2: Matriz de probabilidade de transição nas categorias da chuva mensal abaixo (-1), dentro (0) e acima (1) da climatologia nos municípios utilizados no estudo

\begin{tabular}{|c|c|c|c|c|c|c|c|c|c|}
\hline \multirow{2}{*}{ Categoria } & \multicolumn{3}{|c|}{ Vargem Bonita } & \multicolumn{3}{|c|}{ Saudades } & \multicolumn{3}{|c|}{ Curitibanos } \\
\hline & -1 & $\mathbf{0}$ & 1 & -1 & $\mathbf{0}$ & 1 & -1 & $\mathbf{0}$ & 1 \\
\hline-1 & 0,30 & 0,30 & 0,40 & 0,29 & 0,24 & 0,47 & 0,28 & 0,36 & 0,36 \\
\hline 0 & 0,34 & 0,22 & 0,44 & 0,38 & 0,24 & 0,38 & 0,31 & 0,31 & 0,38 \\
\hline 1 & 0,28 & 0,29 & 0,43 & 0,31 & 0,25 & 0,44 & 0,31 & 0,27 & 0,41 \\
\hline \multirow{2}{*}{ Categoria } & \multicolumn{3}{|c|}{ Lages } & \multicolumn{3}{|c|}{ Trombudo Central } & \multicolumn{3}{|c|}{ Araranguá } \\
\hline & -1 & $\mathbf{0}$ & 1 & -1 & $\mathbf{0}$ & 1 & -1 & $\mathbf{0}$ & 1 \\
\hline-1 & 0,27 & 0,33 & 0,40 & 0,32 & 0,28 & 0,40 & 0,35 & 0,19 & 0,45 \\
\hline 0 & 0,37 & 0,31 & 0,32 & 0,29 & 0,32 & 0,39 & 0,28 & 0,38 & 0,34 \\
\hline 1 & 0,26 & 0,40 & 0,34 & 0,29 & 0,25 & 0,46 & 0,30 & 0,35 & 0,35 \\
\hline \multirow{2}{*}{ Categoria } & \multicolumn{3}{|c|}{ Major Vieira } & \multicolumn{3}{|c|}{ Blumenau } & \multicolumn{3}{|c|}{ Abelardo Luz } \\
\hline & -1 & $\mathbf{0}$ & 1 & -1 & $\mathbf{0}$ & 1 & -1 & $\mathbf{0}$ & 1 \\
\hline-1 & 0,36 & 0,29 & 0,35 & 0,42 & 0,23 & 0,35 & 0,42 & 0,28 & 0,30 \\
\hline 0 & 0,33 & 0,31 & 0,36 & 0,32 & 0,30 & 0,38 & 0,44 & 0,34 & 0,22 \\
\hline 1 & 0,34 & 0,24 & 0,42 & 0,25 & 0,27 & 0,48 & 0,34 & 0,19 & 0,47 \\
\hline
\end{tabular}

Tabela 3: Probabilidade de ter dois $(\mathrm{P}(2))$, um $(\mathrm{P}(1))$ ou zero $(\mathrm{P}(0))$ mês de chuva mensal nas categorias abaixo $(-1)$, dentro $(0)$ e acima (1) da climatologia nos municípios utilizados no estudo

\begin{tabular}{|c|c|c|c|c|c|c|c|c|c|}
\hline \multirow{2}{*}{ Prob. } & \multicolumn{3}{|c|}{ Vargem Bonita } & \multicolumn{3}{|c|}{ Saudades } & \multicolumn{3}{|c|}{ Curitibanos } \\
\hline & -1 & $\mathbf{0}$ & 1 & -1 & $\mathbf{0}$ & 1 & -1 & $\mathbf{0}$ & 1 \\
\hline $\mathrm{P}(2)$ & 0,20 & 0,16 & 0,33 & 0,23 & 0,16 & 0,32 & 0,18 & 0,21 & 0,31 \\
\hline $\mathrm{P}(1)$ & 0,45 & 0,47 & 0,44 & 0,47 & 0,43 & 0,44 & 0,48 & 0,45 & 0,42 \\
\hline $\mathrm{P}(0)$ & 0,34 & 0,37 & 0,20 & 0,30 & 0,41 & 0,22 & 0,34 & 0,34 & 0,27 \\
\hline \multirow{2}{*}{ Prob. } & \multicolumn{3}{|c|}{ Lages } & \multicolumn{3}{|c|}{ Trombudo Central } & \multicolumn{3}{|c|}{ Araranguá } \\
\hline & -1 & $\mathbf{0}$ & 1 & -1 & $\mathbf{0}$ & 1 & -1 & $\mathbf{0}$ & 1 \\
\hline $\mathrm{P}(2)$ & 0,20 & 0,25 & 0,25 & 0,19 & 0,16 & 0,32 & 0,21 & 0,22 & 0,30 \\
\hline $\mathrm{P}(1)$ & 0,47 & 0,49 & 0,47 & 0,48 & 0,51 & 0,42 & 0,42 & 0,41 & 0,47 \\
\hline $\mathrm{P}(0)$ & 0,33 & 0,26 & 0,28 & 0,33 & 0,33 & 0,25 & 0,37 & 0,36 & 0,23 \\
\hline \multirow{2}{*}{ Prob. } & \multicolumn{3}{|c|}{ Major Vieira } & \multicolumn{3}{|c|}{ Blumenau } & \multicolumn{3}{|c|}{ Abelardo Luz } \\
\hline & -1 & $\mathbf{0}$ & 1 & -1 & $\mathbf{0}$ & 1 & -1 & $\mathbf{0}$ & 1 \\
\hline $\mathrm{P}(2)$ & 0,24 & 0,18 & 0,29 & 0,23 & 0,16 & 0,32 & 0,30 & 0,15 & 0,25 \\
\hline $\mathrm{P}(1)$ & 0,44 & 0,42 & 0,44 & 0,39 & 0,42 & 0,42 & 0,44 & 0,50 & 0,38 \\
\hline $\mathrm{P}(0)$ & 0,32 & 0,40 & 0,27 & 0,38 & 0,42 & 0,26 & 0,26 & 0,35 & 0,36 \\
\hline
\end{tabular}


Tendo em mente a categoria de chuva mensal prevista, pode-se recorrer às informações da Tabela 4 para averiguar a probabilidade de ocorrências de eventos extremos diários de chuva. Assim, quando há previsão de a chuva mensal ficar abaixo da climatologia, a probabilidade de não haver evento extremo diário é grande em Santa Catarina (entre 92 e 99\%), bem como, se a previsão for de a chuva ficar dentro da climatologia, a probabilidade da inexistência de evento extremo diário também é maior, sendo predominantemente entre 71 e $84 \%$. A possibilidade de ocorrência de eventos extremos diários de chuva é maior (predominantemente entre 63 e $72 \%$ ), quando a chuva mensal fica acima da climatologia.

Os resultados obtidos mostraram-se importantes ferramentas para uma previsão climática para Santa Catarina mais detalhada e prática, haja vista, que a única informação necessária para sua utilização é o conhecimento da chuva mensal observada e incluí-la em uma das três categorias de chuva (Tabela 1). Muitos modelos estatísticos de previsão climática utilizam a temperatura da superfície do mar (TSM) como dados de entrada, porém, nem sempre para um dado período do ano existe uma correlação significativa com a chuva da região de interesse. Shukla (1998) mostrou que apenas algumas características atmosféricas médias na escala sazonal, sobre determinadas regiões tropicais são fortemente influenciadas pela TSM adjacente. Para o Caribe, Ashby et al. (2005) definiram a TSM do Atlântico e do Pacífico Equatorial como as variáveis com as melhores correlações com a chuva do período chuvoso da região.

Para Santa Catarina, Minuzzi (2010) encontrou boas correlações com até três 'lags' da TSM das quatro regiões Niños no Pacífico Equatorial, com as chuvas de outubro, novembro, abril e maio, no estudo que envolveu os meses de setembro a maio. Cardoso \& Silva Dias (2004) encontraram correlações significativas com até três trimestres de defasagem, entre a TSM do Pacífico e Atlântico com eventos extremos de chuva de regiões pluviometricamente homogêneas do Sul e Sudeste do Brasil, exceto no verão em que o Pacífico apresentou poucas correlações significativas, onde o padrão do Atlântico foi mais representativo.

Tabela 4: Frequência relativa de ocorrência (\%) de eventos extremos (Ee) diários de chuva quando o mês está na categoria -1, 0 ou 1 para os municípios de Vargem Bonita (VB), Abelardo Luz (AL), Saudades (Sau), Curitibanos (Cur), Lages (Lag), Trombudo Central (TC), Araranguá (Ara), Major Vieira (MV) e Blumenau (Blu)

\begin{tabular}{|c|c|c|c|c|c|c|c|c|c|}
\hline & VB & $\mathbf{A L}$ & Sau & Cur & Lag & TC & Ara & MV & Blu \\
\hline Quando categoria -1, não há Ee & 96 & 94 & 99 & 97 & 95 & 96 & 92 & 98 & 96 \\
\hline Quando categoria -1, há 1 Ee & 4 & 6 & 1 & 3 & 5 & 4 & 8 & 2 & 4 \\
\hline Quando categoria 0, não há Ee & 73 & 71 & 83 & 78 & 84 & 64 & 77 & 82 & 81 \\
\hline Quando categoria 0, há $1 \mathrm{Ee}$ & 26 & 27 & 16 & 20 & 15 & 36 & 22 & 18 & 17 \\
\hline Quando categoria 0, há 2 Ee & 1 & 2 & 1 & 2 & 1 & 0 & 1 & 0 & 1 \\
\hline Quando categoria 1, não há Ee & 35 & 31 & 36 & 49 & 37 & 33 & 37 & 34 & 28 \\
\hline Quando categoria 1, há 1 ou + Ee & 65 & 69 & 64 & 51 & 63 & 67 & 63 & 66 & 72 \\
\hline
\end{tabular}

\section{CONCLUSÕES}

Os maiores valores probabilísticos (acima de 45\%) são obtidos para os municípios de Saudades e Araranguá, da categoria abaixo para acima da climatologia, e em Trombudo Central, Blumenau e Abelardo Luz, com a chuva estando acima da climatologia e permanecendo nesta condição no mês seguinte.

As maiores probabilidades para cada uma das três categorias de chuva (abaixo, dentro e acima da climatologia) é que estas ocorram ao menos uma vez a cada dois meses.

Quando as chuvas tendem a ficar abaixo ou dentro da climatologia, a probabilidade de ocorrência de pelo menos um evento extremo diário de chuva é inferior a $30 \%$ em quase todo o estado de Santa Catarina.

\section{REFERÊNCIAS}

ANA - Agência Nacional de Águas (2014) Sistema de Informações Hidrológicas. Disponível em: www.hidroweb.ana.gov.br. Acessado em: 21 de janeiro de 2014.
Ashby SA, Taylor MA \& Chen AA (2005) Statistical models for predicting rainfall in the Caribbean. Theoretical and Applied Climatology, 82:65-80.

Azevedo PV de, Silva BB da \& Rodrigues MFG (1998) Previsão estatística das chuvas de outono no estado do Ceará. Revista Brasileira de Meteorologia, 13:19-30.

Ayoade JO (2003) Introdução à climatologia para os trópicos. Rio de Janeiro, Bertrand Brasil. 332p.

Berlato MA \& Fontana DC (2001) Impacts of El Niño and La Niña on agricultural production in Southern Brazil and use of climate forecasts in agriculture. In: Cunha GR, Hass, JC \& Berlato MA (Eds.) Applications of climate forecasting for better decision-making processes in agriculture. Passo Fundo, Embrapa Trigo. p.217-241.

Braga HJ \& Ghellre R (1999) Proposta de diferenciação climática para o Estado de Santa Catarina. In: $11^{\circ}$ Congresso Brasileiro de Agrometeorologia e $2^{a}$ Reunião Latino-Americana de Agrometeorologia, Florianópolis. Anais, SBAgro. CD-ROM.

Cardoso AO \& Silva Dias PL da (2004) Identificação de trimestres extremos no regime pluviométrico do Sul e Sudeste do Brasil e relação com as anomalias da TSM. Revista Brasileira de Meteorologia, 19:149-162. 
Dias MAFS \& Silva MGAJ (2009) Para entender tempo e clima. In: Cavalcanti IFA, Ferreira NJ, Silva MGAJ da \& Dias MAFS (Eds.) Tempo e clima no Brasil. São Paulo, Oficina de Texto. p.15-21.

Fox G, Turner J \& Gillespie T (1999) The value of precipitation forecast information in winter wheat production. Agricultural and Forest Meteorology, 95:99-111.

Grimm AM (2009) Clima da região Sul do Brasil. In: Cavalcanti IFA, Ferreira NJ, Silva MGAJ da \& Dias MAFS (Eds.) Tempo e clima no Brasil. São Paulo, Oficina de Texto. p.259-275.

IBGE - Instituto Brasileiro de Geografia e Estatística (2014) Banco de dados agregados - Agricultura. Disponível em: <http:// www.sidra.ibge.gov.br/> Acessado: 04 de agosto de 2014.

Minuzzi RB (2010) Chuvas em Santa Catarina durante eventos do El Niño Oscilação Sul. Geosul, 25:107-127.
Soares CPB, Leite HG, Campos JCC \& Sediyama GC (1998) Caracterização da periodicidade e da probabilidade de ocorrência de precipitações anuais como subsídio para o planejamento florestal. Revista Árvore, 22:315-324.

Shukla J (1998) Predictability in the midst of chaos: A scientific basis for climate forecasting. Science, 282:728-731.

Vianello RL \& Alves AR (1991) Meteorologia básica e aplicações. Viçosa, Editora UFV. 449p.

Xavier T de MBS (2001) Tempo de chuva - Estudos climáticos e de previsão para o Ceará e Nordeste Setentrional. Fortaleza, ABC Editora. 478p.

Wilks DS (2006) Statistical methods in the atmospheric sciences. $2^{\mathrm{a}}$ ed. San Diego, Elsevier. 625p. 